\title{
Thrust Bearing Stiffness Testing Based on Multidimensional Loading Device
}

\author{
Guohua Zhao* and Wuyi Chen \\ No.37 Xueyuan Road, Haidian District, Beijing, China \\ *Corresponding author
}

\begin{abstract}
This paper presents a composite 6UPS-6SPS parallel mechanism (PM) based on the Stewart platform for the multidimensional loading test toward the thrust bearing (TB). Analysis such as on the degree of freedom (DoF), kinematic, statics and Jacobian matrix is carried out. Meanwhile, a composite continuous stiffness testing method for the $T B$ without changing the boundary conditions is proposed.
\end{abstract}

Keywords-thrust bearing; stiffness testing; multidimensional loading device; parallel mechanism

\section{INTRODUCTION}

$T B$ is a core component in the rotor system of a helicopter and connects the hub to the flexible blade, providing the wielding, shimmy, and pitch DoF [1, 2]. The stiffness is the main mechanical properties of the $T B$ which is composed of the large joint, small joint and the rubber-metal laminated structure [3]. Although compression, torsion and bending tests were carried out respectively by Huang [4], the results might not be accurate enough for these were obtained by the uniaxial and biaxial stiffness testing rather than by the complex loads in the actual working conditions. The properties of material vary according to the loading order and loading value, that's to say the different loading orders and values can result in the coupling effect [5, 6, 7]. A novel Stewart platform-based manipulator was analyzed for applications where ultrahigh accurate positioning is required under large external loads by Ding [8]. A multidimensional loading material testing machine was proposed and multidimensional loading and standard tension material tests were established by Guo [9-11]. These researches showed that a loading equipment in the parallel mechanism format could be adopted to apply multidimensional load simultaneously.

In this paper, a Stewart platform-based multidimensional loading device, 6UPS-6SPS PM, is proposed for the stiffness testing of the $T B$. Since the testing device possesses six $D o F$, the stiffness of the $T B$ can be obtained in the multidimensional loading situation where the properties of the $T B$ is different from the state of the uniaxial and biaxial stress.

\section{KINEMATIC DESCRIPTION}

The CAD model of the composite 6UPS-6SPS PM is shown in Figure 1 and the schematic diagram is described by Figure 2.

The testing device consists of a 6UPS PM, a 6SPS PM and a fixed frame. The 6UPS PM is the multidimensional loading device, and is composed of six motor-driven kinematic limbs, a moving platform assembly at the top, and a supporting frame at the bottom. Each limb which is driven by a ball screw actuator connects the fixed frame to the moving platform with a spherical (S) joint followed by a prismatic joint $(\mathrm{P})$ and a universal (U) joints. The TB is mounted between the lower and upper fixture. The lower grip is connected to the fixed frame via a height adjustable pillar and a 6DOF dynamometer which is used to measure the forces and moments applied to the thrust bearing while the upper grip is directly mounted to the moving platform by another height adjustable pillar. Based on the Stewart platform design, the top 6SPS PM mainly consists of six telescopic rods with grating, which share the moving platform with the 6UPS PM and use a separated supporting frame connected to the top of the fixed frame

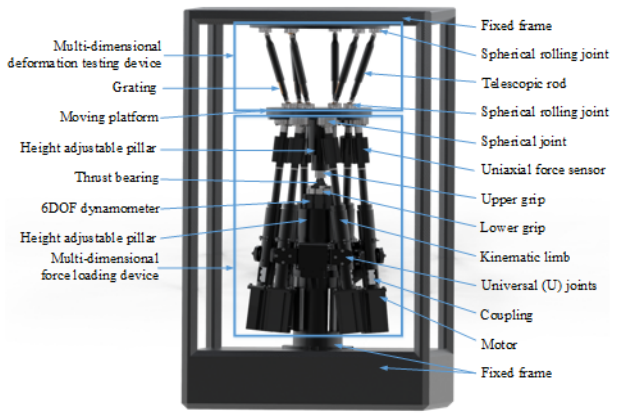

FIGURE I. THE CAD MODEL OF THE 6UPS-6SPS PM

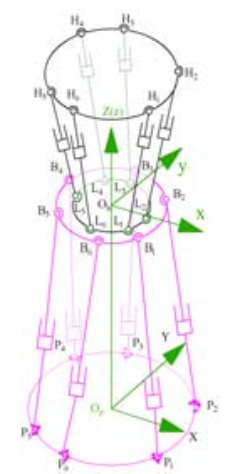

FIGURE II. THE SCHEMATIC DIAGRAM 6UPS-6SPS PM

The DoF solution which is used to determine the capability of the mechanism is given in Eq. 1. The details of the DoF solution is introduced in [12]. 


$$
M=6(n-g-1)+\sum_{1}^{i} f_{i}
$$

where $\mathrm{n}$ and $\mathrm{g}$ are, respectively, the number of bodies and the number of joints of the $P M$, and $f_{i}$ is the number of freedoms of the joint $i$. Given the particular topological structure of the mechanism, we have $\mathrm{b}=26, \mathrm{~g}=36$ and $\sum_{1}^{i} f_{i}=72$, the mobility $\mathrm{M}$ of the mechanism is six.

\section{STATICS ANALYSIS AND JACOBIAN MATRIX}

Since statics analysis of the 6UPS mechanism is similar with the 6SPS mechanism, only the statics analysis of the 6SPS mechanism is researched here via the screw theory.

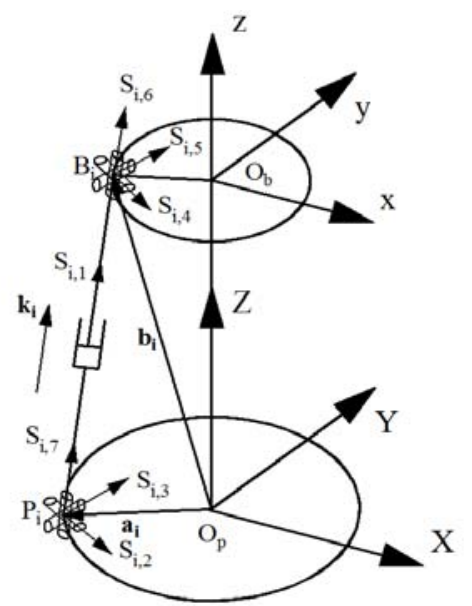

FIGURE III. EQUIVALENT MODEL OF THE ITH LIMB

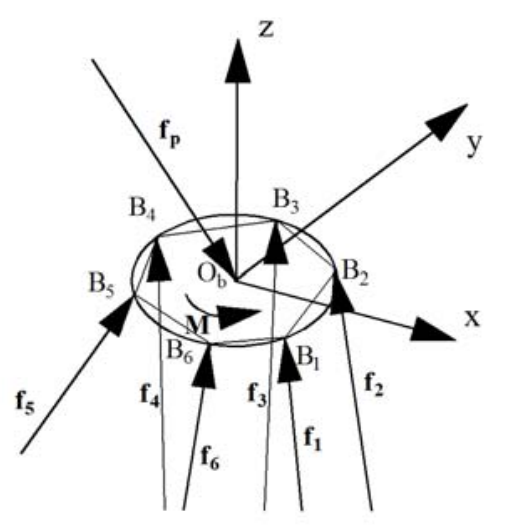

FIGURE IV. FORCE DIAGRAM OF THE MOVING PLATFORM

The spherical joint works in the same way as three revolute joints with orthogonal axes so that each limb of the 6SPS structure can be kinematically equivalent to a PRRRRRR leg as shown in Figure 3. Figure 4 is the forces and moments acting on the moving platform.
As shown in Figure 3, the spherical joint $P_{i}$ is substituted by three revolute joints with three orthogonal unit axis vectors $\overrightarrow{\mathbf{S}}_{i, 2}$, $\overrightarrow{\mathbf{S}}_{i, 3}, \overrightarrow{\mathbf{S}}_{i, 7}$. Vector $\overrightarrow{\mathbf{S}}_{i, 7}$ lies along the axis of the leg $P_{i} B_{i}$. Vector $\overrightarrow{\mathbf{S}}_{i, 2}$ is perpendicular to vector $\overrightarrow{\mathbf{S}}_{i, 7}$ while vector $\overrightarrow{\mathbf{S}}_{i, 3}$ can be derived by the right-hand rule. The spherical joint $B_{i}$ can be similarly decomposed to three revolution joints, respectively, with three unit axis vectors $\overrightarrow{\mathbf{S}}_{i, 4}, \overrightarrow{\mathbf{S}}_{i, 5}$ and $\overrightarrow{\mathbf{S}}_{i, 6}$. Vector $\overrightarrow{\mathbf{S}}_{i, 6}$ coincides with $\overrightarrow{\mathbf{S}}_{i, 7}$ while vector $\overrightarrow{\mathbf{S}}_{i, 4}$ and $\overrightarrow{\mathbf{S}}_{i, 5}$ are parallel to vector $\overrightarrow{\mathbf{S}}_{i, 2}$ and $\overrightarrow{\mathbf{S}}_{i, 3}$ respectively. As the rotations about the vector $\overrightarrow{\mathbf{S}}_{i, 6}$ and $\overrightarrow{\mathbf{S}}_{i, 7}$ exert the same influence on the ith limb, they are both indicated by the unit vector $\overrightarrow{\mathbf{k}}_{i}$, which is along each limb in the following analysis. $\overrightarrow{\mathbf{b}}_{i}$ indicates the vector $\overrightarrow{\mathbf{O}}_{P} \mathbf{B}_{i}$. It should be noted here that all of the vectors mentioned in this paper is in the fixed Cartesian reference frame $O_{p}[\mathrm{X}, \quad \mathrm{Y}, \mathrm{Z}]$.Assume that an external wrench $\overrightarrow{\mathbf{F}}=\left[\begin{array}{ll}\overrightarrow{\mathbf{f}}_{p}{ }^{T} & \overrightarrow{\mathbf{M}}^{T}\end{array}\right]^{T}$ expressed in the Plücker coordinate is acted on the mobile platform. As shown in Figure 4, $\overrightarrow{\mathbf{f}}_{i}$ represents the force vectors along each limb respectively for $i=1$ to $6, \overrightarrow{\mathbf{f}}_{p}$ denotes the external force vector while $\overrightarrow{\mathbf{M}}$ indicates the torque vector acting on the moving platform. The instantaneous wrench of the mobile platform can be expressed as Eq. 2.

$$
f_{1} \$_{1}+f_{2} \$_{2}+f_{3} \$_{3}+f_{4} \$_{4}+f_{5} \$_{5}+f_{6} \boldsymbol{\$}_{6}=\left(\overrightarrow{\mathbf{f}}_{\mathrm{P}}^{\mathrm{T}}, \overrightarrow{\mathbf{M}}^{\mathrm{T}}\right)^{\mathrm{T}}
$$

For $\mathrm{i}=1$ to $6, f_{i}$ denotes the value of the $\mathbf{f}_{i}, \boldsymbol{\$}_{i}$ indicates the unit screw associated with the ith limbs. Eq. 2 may be expressed in a matrix form as

$$
\overrightarrow{\mathbf{F}}=\mathbf{J}_{\mathrm{f}} \overrightarrow{\mathbf{f}}
$$

where $\quad \mathbf{J}_{\mathrm{f}}=\left(\begin{array}{llllll}\boldsymbol{S}_{1} & \boldsymbol{\$}_{2} & \boldsymbol{S}_{3} & \boldsymbol{\$}_{4} & \boldsymbol{S}_{5} & \boldsymbol{\$}_{6}\end{array}\right)$ is the Jacobian matrix, $\quad \overrightarrow{\mathbf{f}}=\left(\begin{array}{llllll}f_{1} & f_{2} & f_{3} & f_{4} & f_{5} & f_{6}\end{array}\right)^{\mathrm{T}} \quad$ and $\boldsymbol{S}_{i}=\left(\overrightarrow{\mathbf{S}}_{i} ; \overrightarrow{\mathbf{S}}_{o, i}\right) . \overrightarrow{\mathbf{S}}_{i}$ which is equal to $\overrightarrow{\mathbf{K}}_{i}$ indicates the unit vector along each limb, while $\overrightarrow{\mathbf{S}}_{o, i}$ represents the moment of line about $\overrightarrow{\mathbf{S}}_{i}$ with respect to the fixed reference frame $O_{p}$. According to the vector operation, $\overrightarrow{\mathbf{S}}_{i}$ and $\overrightarrow{\mathbf{S}}_{o, i}$ can be described as 


$$
\begin{aligned}
& \overrightarrow{\mathbf{S}}_{i}=\frac{{\overrightarrow{\mathbf{O}_{\mathrm{p}} \mathbf{B}_{i}}}_{i}-{\overrightarrow{\mathbf{O}_{\mathrm{p}}}}_{i}}{\left|\overrightarrow{\mathbf{O}_{\mathrm{p}} \mathbf{B}_{i}}-\overrightarrow{\mathbf{O}_{\mathrm{p}} \overrightarrow{\mathbf{P}}_{i}}\right|} \\
& \overrightarrow{\mathbf{S}}_{o, i}=\overrightarrow{\mathbf{b}}_{i} \times \overrightarrow{\mathbf{S}}_{i}
\end{aligned}
$$

Substituting Eq. 4 and Eq. 5 in Eq. 3 yields

$$
\mathbf{J}_{\mathrm{f}}=\left(\begin{array}{cccc}
\overrightarrow{\mathbf{k}}_{1} & \overrightarrow{\mathbf{k}}_{2} & \cdots & \overrightarrow{\mathbf{k}}_{6} \\
\overrightarrow{\mathbf{b}}_{1} \times \overrightarrow{\mathbf{k}}_{1} & \overrightarrow{\mathbf{b}}_{2} \times \overrightarrow{\mathbf{k}}_{2} & \cdots & \overrightarrow{\mathbf{b}}_{6} \times \overrightarrow{\mathbf{k}}_{2}
\end{array}\right)_{6 \times 6}
$$

Since the gravity of the limbs contributes to the Jacobian matrix, it needs to be taken into account. Assume that the gravity of each limb is $\overrightarrow{\boldsymbol{G}}_{\boldsymbol{i}}$. For i $=1$ to 6 , the gravity projection of each limb along $\overrightarrow{\mathbf{k}}_{i}$ can be expressed as

$$
f_{g i}=\overrightarrow{\boldsymbol{G}}_{i} \cdot \overrightarrow{\boldsymbol{l}}_{\boldsymbol{i}}
$$

Substituting Eq. 7 in Eq. 2 yields

$$
\left(f_{1}+f_{g 1}\right) \$_{1}+\left(f_{2}+f_{g 2}\right) \$_{2}+\left(f_{3}+f_{g 3}\right) \$_{3}+\left(f_{4}+f_{g 4}\right) \$_{4}+\left(f_{5}+f_{g 5}\right) \$_{5}+\left(f_{6}+f_{g 6}\right) \$_{6}=\left(\overrightarrow{\mathbf{f}}_{\mathrm{P}}^{\mathrm{T}}, \overline{\mathbf{M}}^{\mathrm{T}}\right)^{\mathrm{T}}
$$

Eq. 8 may be expressed in a matrix form as

$$
\mathbf{J}_{\mathrm{f}} \overrightarrow{\mathbf{F}}^{\prime}=\left(\overrightarrow{\mathbf{f}}_{\mathbf{P}}^{\mathrm{T}}, \overrightarrow{\mathbf{M}}^{\mathrm{T}}\right)^{\mathrm{T}}
$$

where $\overrightarrow{\mathbf{F}}=\left(f_{1}+f_{g 1}, f_{2}+f_{g 2}, f_{3}+f_{g 3}, f_{4}+f_{g 9}, f_{5}+f_{g,}, f_{6}+f_{g 6}\right)^{\mathrm{T}}$

\section{STIFFness Testing Method}

Since the composite 6UPS-6SPS PM possesses six DoF, the $T B$ can be loaded with three dimensional forces and three moments at the same time. Meanwhile, the multidimensional deformation testing device can measure the deformation of the $T B$ through the forward kinematics of 6SPS mechanism using the displacements gauged by the gratings on each telescopic rod. Here, we propose a composite stiffness testing method through which more realistic properties of the $T B$ can be obtained compared with the conventional method.

Previous work of us have shown that the method of using the $P M$ to apply multidimensional loading to an elastic specimen is feasible [9]. Therefore, according to the material multidimensional loading experiment we propose a $T B$ multidimensional loading method through which the $T B$ properties can be measured and researched in the complex stress state that is much more consistent with the actual runtime situation.

The uniaxial compression stiffness testing method is described in Figure 5 as an example of the conventional method and a composite stiffness testing method is mentioned in Figure 6 as well. In order to obtain more realistic stiffness of the TB in the actual working conditions, a composite stiffness testing method is proposed to meet the various stiffness testing toward the TB. The certain loading forces which are used in the conventional stiffness testing method are substituted by the general values and different kinds of loading forces in the composite stiffness testing method.

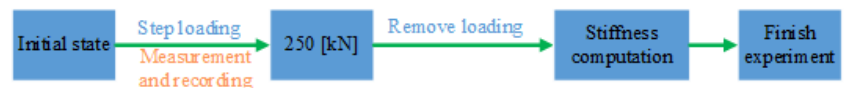

FIGURE V. UNIAXIAL COMPRESSION STIFFNESS TESTING OF THE CONVENTIONAL METHOD

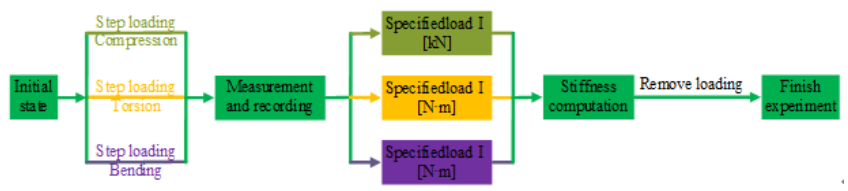

FIGURE VI. COMPOSITE STIFFNESS TESTING METHOD

As can be seen from the two figures above, for the conventional stiffness testing method of the TB only the uniaxial compression is considered while the compression, torsion and bending as well as their values are all taken into account in the composite stiffness testing method. During the composite stiffness testing, the three kinds of loads can be arranged with different values which are closer with the actual situation. The loads acted on the TB can be directly measured through the $6 \mathrm{DoF}$ dynamometer mounted on the supporting frame. The forces along each limb can be solved from the Eq. 9

$$
\overrightarrow{\mathbf{f}^{\prime}}=\mathbf{J}_{\mathrm{f}}^{-1}\left(\frac{\overrightarrow{\mathbf{f}}_{p}}{\overrightarrow{\mathbf{M}}}\right)-\overrightarrow{\mathbf{f}}_{g}
$$

where $\overrightarrow{\mathbf{f}}_{g}=\left(f_{g 1}, f_{g 2}, f_{g 3}, f_{g 4}, f_{g 5}, f_{g 6}\right)^{T}$ is the vector form of the $f_{g i}$.

For $\mathrm{i}=1$ to $6, f_{g i}$ can also be measured via the six uniaxial force sensors on each limb. $f_{g i}$ obtained from the two different methods can be used to guarantee the correctness of loading experiment.

\section{SUMMARY}

Based on our previous PM loading test device for metallic material, a precision 6UPS-6SPS PM device which is used for the stiffness testing of the helicopter TB under the multidimensional loading is proposed. The architecture of the 6UPS-6SPS PM is presented in detail. Meanwhile, the DoF 
analysis, statics analysis and Jacobian matrix are carried out based on the screw theory. Finally, according to the 6UPS-6SPS PM, a composite stiffness testing method for the TB is proposed in the complex stress state.

\section{REFERENCES}

[1] M. F. Li, J. T. Cheng and Q. L. Wang, Review of the Rotor Hub Configuration Technology, J. Helicopter Technique. 04 (2010) 64-70.

[2] J. Wang, R. F. Qin and Y. X. Wu, Spherical Thrust Bearings Load Spectra Adjust Based on Flight Test and Service Life Evaluation, J. Helicopter Technique. 01 (2013) 1-3.

[3] H. Y. Qin, L. X. Jia and H. G. Yang, The Failure Influence Analysis of Helicopter Rotor Elastomeric Spherical Thrust Bearing, J. Helicopter Technique. 04 (2011) 16-20.

[4] X. D. Huang, W. W. Wang and W. G. Jiang, Research of Stiffness Characteristics Test Method for Helicopter Rotor System Spherical Thrust Bearing, J. Mechanical Strength. 34 (2012) 270-273.

[5] S. Sen and S. Awtar, Nonlinear Constraint Model for Symmetric Three-Dimensional Beams, Amer Soc Mechanical Engineers, New York, 2010.

[6] S. Sen and S. Awtar, Nonlinear Strain Energy Formulation of a Generalized Bisymmetric Spatial Beam for Flexure Mechanism Analysis, J. Sci. Mechanical Design. 136 (2014) 165-173.

[7] D. H. D. Hodges and E. H. Dowell, Nonlinear Equations of Motion for the Elastic Bending and Torsion of Twisted Nonuniform Rotor Blades, U.S Army Air Mobility Research and D. L. A. R. Center, California, 1974.

[8] B. Ding, B. S. Cazzolato, R. M. Stanley, S. Grainger and J. J. Costi, Stiffness Analysis and Control of a Stewart Platform-Based Manipulator with Decoupled Sensor-Actuator Locations for Ultrahigh Accuracy Positioning Under Large External Loads, J. Sci. Dynamic Systems Measurement \& Control. 136 (2014).

[9] J. Z. Guo, D. Wang, R. Fan, W. Y. Chen and G. H. Zhao, submitted to Journal of Advanced Mechanical Design, Systems, and Manufacturing (2016).

[10] D. Wang, R. Fan, J. Z. Guo, W. Y. Chen and Y. S. Sun, Design and Experimental Study of a 6-DOF Material Testing Machine, J. Applied Mechanics \& Materials Applied Mechanics \& Materials. 419 (2013) 533-542.

[11] J. Z. Guo, R. Fan, D. Wang, W. Y. Chen and Y. S. Sun, Development of Multidimensional Loading Material Testing Machine, J. Materials Science Forum. 770 (2014) 164-169.

[12] Z. Huang, Y. S. Zhao and T. S. Zhao, Advanced Spatial Mechanism, Higher Education Publication, Beijing, 2006. 